\title{
Quelle importance a l'environnement ? Enseignements du cas Lomborg
}

\author{
Edwin Zaccaï $^{\mathrm{a}, \star}$, François Goor ${ }^{\mathrm{b}}$, Bruno Kestemont ${ }^{\mathrm{c}}$ \\ a Ingénieur et philosophe, Université Libre de Bruxelles, Centre d'études du développement durable (IGEAT), CP 130/02, \\ Avenue F. Roosevelt, 50, 1050 Bruxelles, Belgique \\ b Agronome, Université Libre de Bruxelles, Centre d'études du développement durable (IGEAT), CP 130/02, Avenue F. Roosevelt, \\ 50, 1050 Bruxelles, Belgique \\ c Statisticien, Université Libre de Bruxelles, Centre d'études du développement durable (IGEAT), CP 130/02, Avenue F. Roosevelt, \\ 50, 1050 Bruxelles, Belgique
}

\section{Mots-clés :}

environnement; indicateurs ; croissance; pollution; air

\section{Keywords:} environment; indicators; growth; pollution; air
Résumé - The Skeptical Environmentalist. Measuring the Real State of the World, écrit en 18 mois par le statisticien Bjorn Lomborg, est devenu un ouvrage bien connu dans le monde, par l'importance de ses ventes et des polémiques qu'il suscite. Ce livre entend combattre, avec une verve ironique et beaucoup de données chiffrées, la « litanie » environnementaliste (médias, organisations et scientifiques assimilés) qui selon lui assombrit à tort l'évolution favorable du développement mondial vers le progrès. Des erreurs factuelles ont cependant été constatées, dont certaines pourraient n'être pas entièrement involontaires. Le rapprochement entre les faits et la thèse avancée semble s'appuyer sur un choix et un ordonnancement des données très contestables. Cet article en donne une illustration par l'examen du problème de la déforestation et de celui du rejet de polluants acidifiants dans l'air. Le cas Lomborg permettra de s'interroger sur les raisons de la fixation des débats écologiques en termes d'oppositions manichéennes et sur les leçons que l'on peut tirer de son retentissement.

\begin{abstract}
How much does environment matter? Lessons from the Lomborg case. The Skeptical Environmentalist. Measuring the Real State of the World, written under 18 month by statistician Bjorn Lomborg, has become famous in the world due to its significant sales and the polemics it aroused. Lomborg's book intends to fight, with ironical eloquence and plenty of figures, the environmentalist "litany" (carried by media, organisations and scientists) which according to him wrongly blackens the favourable evolution of the world development toward progress. Yet as far as figures are concerned, many specialists have already pointed out different errors, some of which might not be entirely involuntary. Anyway, one can but observe the strange regularity with which facts confirm the arguments maintained and the fact that the data are often selected accordingly. The present article illustrates this observation by the specific study of the treatment of the problems of deforestation and acid air pollution. In the case of deforestation, the apparent stability of the forest surface is only apparent when one aggregates disparate, uncertain data, for all the Earth regions together, and above all neglecting differences of forest types value. In the case of air pollution, the relative and partial reduction which takes place at some stages of economic growth can neither be generalised (at global level this pollution increases), nor be considered spontaneous, even though the author suggests a spontaneist view of the improvements brought about by growth and technologies. Other presuppositions and problem framings by Lomborg are analysed in this paper with a view to understanding the reasons for fixing ecological debates in terms of dualistic oppositions. We also suggest some lessons to be drawn from the considerable influence of that case.
\end{abstract}

Depuis la mi-2001, un livre a alimenté d'importantes controverses à propos du "véritable état de la planète » et, chose encore plus sérieuse, de la compatibilité du

^ Auteur correspondant : ezaccai@ulb.ac.be développement actuel avec l'environnement. Ce livre, c'est The Skeptical Environmentalist. Measuring the Real State of the World, un ouvrage de 515 pages, y compris 2930 notes, écrit en 18 mois par Bjorn Lomborg, un universitaire danois spécialisé en statistiques. 
Encadré. Recension d'articles ayant alimenté ou commenté le débat sur Lomborg dans des revues scientifiques (2001-2003) (arrêtée en septembre 2003).

Bongaarts, J., 2002. Population: ignoring its impact, Scientific American, 286, 6769.

Budiansky, S., 2002. Divisionary tactics in environmental debate, Nature, 415, 364.

Burgman, M., 2002. The Skeptical Environmentalist. Measuring the Real State of the World, Austral Ecology, 27, $238-239$.

Chadwick, M.J., 2002. The Skeptical Environmentalist. Measuring the Real State of the World, Climate Policy, 2, $259-261$.

Cole, M.A., 2003. Environmental optimists, environmental pessimists and the real state of the world: An article examining The skeptical environmentalist: Measuring the real state of the world by Bjorn Lomborg, The Economic Journal, 113, $362-380$.

Czech, B., 2000. Julian Simon Redux, Conservation Biology, 16, 2, 570-571.

Dagg, J.L., 2003. The Skeptical Environmentalist and the Lomborg Affair, Environmental Science E Policy, 6, 4, sous presse.

Godard, O., 2002. Bjorn Lomborg ou Tintin au pays de l'écologie? Une revue critique du livre de Bjorn Lomborg, The Skeptical Environmentalist, Politique étrangère, IFRI.

Grubb, M., 2001. Relying on Manna from Heaven? Science, 294, 1285-1286.

Holdren, J., 2002. Energy: asking the wrong questions, Scientific American, 286, 6567.

Lovejoy, T., 2002. Biodiversity : dismissing scientific process, Scientific American, 286, 1, 6971.

Moomaw, W.R., 2000. Lomborg's The Skeptical Environmentalist: Refuting a Scientific Model without Science, Conservation Biology, $16,4,861-862$.

Orr, D.W., 2002. The Labor of Sisyphus, Conservation Biology, 16, 4, 857-860.

Pauly, D., 2002. The Skeptical Environmentalist. Measuring the Real State of the World, Fisheries, 3, 2002, 360-365.

Pielke Jr., R.A., 2002. Policy, politics and perspective, Nature, 416, 367-368.

Pimm, S., Harvey, J., 2001. No need to worry about the Future, Nature, 414, 149-150.

Ridley, M., 2002. The profits of doom, Spectator, 288, 1011.

Schneider, S., 2002. Global warming: neglecting the complexities, Scientific American, 286, 6265.

À quoi on ajoutera la publication copieuse réalisée par certains de ses collègues danois :

Ege, C., Lind Christiansen, J., 2002. Sceptical Questions and Sustainable Answers, Danish Ecological Council, Copenhaguen.

Cette affaire dépasse la simple recension d'un ouvrage pour plusieurs raisons. Outre le tirage important pour ce genre d'ouvrage, largement plus de 100000 exemplaires dans sa version anglaise, et sa traduction en plusieurs langues, la figure de Lomborg a été mise en avant dans d'importants journaux anglo-saxons et autres - en particulier danois, pays dont l'auteur est originairecomme un point focal majeur de contestation d'un certain catastrophisme environnemental, et finalement des politiques environnementales elles-mêmes. Les polémiques, qui n'ont pas manqué, ont porté d'abord sur la fiabilité des évaluations réalisées. Mais, au-delà, elles interrogent les règles du travail scientifique et les rapports de celui-ci avec la formation d'opinions et de décisions. C'est pourquoi nous voudrions revenir sur la présentation des éléments de cette affaire et sur certaines leçons qu'elles permettent de tirer, au-delà de ce livre emblématique ${ }^{1}$.

\section{Controverses scientifiques}

Le livre de Lomborg est construit selon une visée essentiellement polémique. Publié chez un éditeur universitaire, Cambridge University Press, il se situe dans une collection consacrée aux sciences sociales, de sorte qu'il

\footnotetext{
${ }^{1}$ L'origine de cet article se situe dans un séminaire de doctorants et post-doctorants, dont plusieurs travaillent sur les indicateurs de développement durable, tenu en février 2003 à l'Institut de gestion de l'environnement et d'aménagement du territoire de l'Université Libre de Bruxelles, et non publié.
}

n'a apparemment pas subi de peer review portant sur la fiabilité des données relatives aux sciences naturelles, qui pourtant forment l'essentiel du dossier instruit à charge des environnementalistes. Disons tout de suite que cette lacune aura une portée d'autant plus grande qu'elle s'ajoute au fait que l'auteur se déclare ouvertement non spécialiste de l'environnement, et qu'à toutes les critiques factuelles qui lui seront faites par des experts confirmés des problématiques dont il traite, il répondra par un déni assez généralisé.

Aujourd'hui, la lecture des écrits de toutes sortes que l'ouvrage a suscités ${ }^{2}$ fait apparaître un contraste assez net entre des appréciations issues de journaux d'information, et d'autres formulées par une série de scientifiques (Encadré ci-dessus). " $C^{\prime}$ est un des livres les plus précieux sur la politique publique - pas seulement la politique environnementale - qui ait été écrit pour le lecteur général intelligent ces dix dernières années [...]. The Skeptical Environmentalist est un triomphe », estime par exemple The Economist. " C'est le travail le plus significatif sur l'environnement depuis la parution de son pôle opposé, Silent Spring, de Rachel Carson en 1962. Il s'agit d'un accomplissement magnifique », évalue le Washington Post Book World $^{3}$.

À l'inverse, une série d'articles dans des revues telles Nature, Science, Climate Policy, ou Conservation Biology

\footnotetext{
${ }^{2}$ Dont on trouvera une partie sur les sites pro http://www.lomborg.com/ et contra Lomborg http://www.mylinkspage.com/lomborg.html 3 Cités par l'éditeur.
} 
vont critiquer, selon des modalités diverses, des erreurs parfois si nombreuses sur des points cruciaux que l'intention, le fondement et la méthode de l'ouvrage en deviennent troublants. Néanmoins, certains de ces articles reconnaissent aussi des mérites d'indépendance et de sens critique à Lomborg, et une revue comme The New Scientist estime par exemple qu'il s'agit "sûrement du livre le plus controversé et le plus influent sur l'environnement de ces derniers temps ».

Scientific American a donné l'occasion à quatre experts reconnus de revoir les analyses effectuées par Lomborg dans plusieurs chapitres. Chacun met en évidence certaines erreurs manifestes dues à la sélection de sources opportunes, au traitement des données, ou à la méconnaissance des phénomènes analysés. Ainsi pour le climat, Stephen Schneider relève des éléments erronés en matière de connaissance de science du climat, de scénarios d'émissions, et surtout de calculs coûts-bénéfices, un sujet crucial qui parcourt tout l'ouvrage, comme on le verra. Dans sa réponse à ces critiques, dans la même revue, Lomborg ne consent pas à revoir ses résultats. Constatant qu'il est impossible de dialoguer de cette façon, John Holdren, l'un de ses contradicteurs, remarque : «Déformation de ce que j'ai écrit, obscurcissement de ce qu'il a écrit; confusions conceptuelles persistantes, manque de précision là où la spécificité était requise; précision illusoire où seules des approximations sont possibles ${ }^{4}$. »

Faut-il donc brûler Lomborg? Pas si vite.

\section{Démanteler la litanie}

Si les deux «camps » que nous avons schématisés ci-dessus peuvent en effet s'observer, il existe aussi une large frange de commentateurs dans la presse généraliste qui ont bien saisi la portée idéologique de ce livre, à savoir l'occasion de relancer avec force le débat sur une vision alarmiste des impacts environnementaux du développement. C'est le cas par exemple en France avec Libération, Courrier international, ou Le Monde, lequel, fait assez rare, recense dans son édition du Monde des Livres cet ouvrage en anglais, précisément pour sa vertu interpellante ${ }^{5}$.

Le livre est très bien écrit et bien présenté. Sa structure sous forme d'enquête, son style dynamique et polémique, la multiplication des informations qu'il présente, le rendent attractif. Dès les premières pages le ton est donné. Il s'agit de passer au crible des données factuelles et statistiques, la vision vert sombre des éditions annuelles de L'état du monde, la publication phare du Worldwatch Institute. Les grandes associations de protection de

\footnotetext{
${ }^{4}$ Les éléments de la polémique dans le Scientific American sont tirés du jugement du Danish Committee on Scientific Dishonesty. La référence des articles figure dans l'encadré.

5 Dans L'Express (22 août 2002) en revanche, Claude Allègre est unanimement louangeur.
}

l'environnement, mais aussi certains organismes publics ainsi que les scientifiques actifs dans le domaine de l'environnement sont interpellés. Tous sont peu ou prou assimilés à un discours «environnementaliste » multipliant les poncifs sur les conséquences négatives de notre mode actuel de développement : «la litanie ». L'enquête commence par des données portant notamment sur la démographie et la faim dans le monde, ce sont sans doute les chapitres les moins critiqués du livre (surtout le premier). Ils rejoignent les affirmations du Programme des Nations unies pour le développement (PNUD) pour lequel les progrès dans certains aspects du développement humain ont été en effet considérables au XXe siècle, surtout s'ils sont évalués globalement.

La plupart des problématiques environnementales sont ensuite analysées à travers une série de sources et de raisonnements choisis, de telle sorte qu'invariablement les conclusions montrent que soit le problème n'existe pas, c'est un artefact (exemple de la diminution de la fertilité masculine), soit il a été grossièrement surestimé (cas de la diminution de la superficie des forêts ou de la biodiversité), soit les mesures prises ou préconisées pour le combattre sont excessivement coûteuses, mesurées à une utilisation efficiente des ressources économiques (exemple du climat).

Ce qui vaut une conclusion dans les termes suivants: «donc, voici le vrai message du livre : les enfants nés aujourd'hui - tant dans le monde industrialisé que dans les pays en développement - vivront plus longtemps et en meilleure santé. Ils recevront plus de nourriture, une meilleure éducation, un plus haut standard de vie, plus de temps de loisirs et bien plus de possibilités sans que l'environnement global ne soit détruit. Et cela, c'est un monde magnifique. »

Pour Lomborg, le développement économique et technique porte en lui ses propres remèdes. Les impacts sont sous contrôle, ils restent relativement mineurs, ou s'atténueront grâce aux innovations technologiques. Le progrès technique, allié à différentes autres formes d'améliorations (économique, sociale, et même environnementale) est réhabilité, alors qu'il avait été contesté et que des alternatives sont recherchées au moins depuis le dernier quart de siècle. La culpabilité nourrie par les «environnementalistes » est inutile. Lomborg estime que la litanie, relayée complaisamment par les médias, est le fruit du goût de sensationnel du public et de la recherche de notoriété et de crédits par ceux qui la portent ${ }^{6}$. Mieux encore, il y a bien des grandes causes à défendre dans le développement, en particulier celles de l'amélioration de la vie des plus défavorisés, et Lomborg se fait l'avocat

\footnotetext{
${ }^{6}$ Étrangement, et comme le relèvent plusieurs commentateurs, Lomborg ne dit mot des soutiens d'acteurs économiques, telle l'industrie pétrolière, à des études ou des campagnes de ton opposé, pas plus qu'il ne cite des lobbies industriels.
} 
d'actions vers ces priorités-là, plutôt que vers les priorités environnementales.

Par ces quelques éléments de présentation, on conçoit vite combien les discussions peuvent nous emmener loin. Le livre a réussi à condenser et à porter à l'avant-scène des éléments centraux des conceptions actuelles du développement durable. Mais s'il a pu jouer ce rôle de point focal des débats, c'est aussi parce qu'il se présente comme basé sur «la science », qu'il prétend faire acte de libre pensée, informée par la raison et l'observation, opposée à ce dogmatisme ambiant que serait devenu le progressisme écologiste. Sans cette abondante «armure scientifique», il aurait été placé sur le même rang qu'une série d'ouvrages d'opinion. Pourtant, nous avons noté déjà que la scientificité de nombre des résultats présentés a été contestée. Dans ce qui suit, nous proposons de considérer un peu plus en détail deux thèmes où l'on pourra mieux juger du type de traitement effectué à l'appui des thèses défendues : le problème de la déforestation et celui de la pollution de l'air.

\section{Perdu dans les forêts}

En ce qui concerne les forêts, l'essentiel de l'argumentation de Lomborg (chapitre 10) porte sur l'évolution récente de la couverture forestière mondiale, l'objectif étant de démontrer que, contrairement aux slogans de la litanie, elle ne diminuerait pas de façon inquiétante. Et en effet, les séries temporelles disponibles (provenant de l'Organisation des Nations Unies pour l'alimentation et l'agriculture, acronyme anglais : FAO) ne permettent pas de mettre en évidence une évolution significative au cours de la seconde moitié du XXe siècle.

Les données exprimées en termes relatifs (en proportion d'occupation du sol) ne donnent cependant pas une idée claire de l'ampleur des variations. De plus, l'interprétation qui en est faite par Lomborg, ainsi que le peu d'intérêt accordé à l'incertitude qui leur est associée, posent question de la part d'un statisticien. Le choix de longues séries temporelles se justifie, selon Lomborg, par la volonté de mettre en évidence une tendance de fond en lissant les variations à court terme. Dans son interprétation, il gomme néanmoins du même coup les effets significatifs des changements de nomenclature ${ }^{7}$ ou des modes de calcul, ainsi que ceux de l'évolution des techniques de mesure ${ }^{8}$. L'argument de l'incertitude peut donc aisément se retourner contre l'auteur. Dans le même ordre d'idées,

\footnotetext{
7 La définition de la « forêt», basée sur la projection au sol de la couverture du feuillage (canopée), a été récemment modifiée dans le cas des forêts tropicales.

8 Telles que l'utilisation de l'imagerie satellite. Cette évolution a notamment mené à l'abandon, en 1994, d'une des séries de données présentées par Lomborg, pour cause de trop grandes imprécisions.
}

une analyse critique des informations relatives aux dégâts des feux de forêts suggère une utilisation orientée des sources, qui va dans le sens de la thèse générale défendue tout au long de l'ouvrage.

Mais ce n'est peut-être pas le problème fondamental. Même en admettant que, globalement, la couverture forestière mondiale ne diminue pas ou peu, les variations locales ne sont pas ou peu quantifiées. Bien que conscient de la difficulté de comparer différents types de forêts, Lomborg n'hésite pas à les agréger pour l'analyse. Ce faisant, il met sur pied d'égalité une forêt primaire tropicale et une plantation mono-spécifique en zone tempérée. Même si on peut en discuter, un large consensus existe à propos des différences de richesse biologique (biodiversité) et d'intérêt patrimonial (valeur de conservation). Dans ce contexte, une analyse plus détaillée montre que la relative stabilité au niveau mondial au cours des dernières décennies masque en réalité une augmentation des forêts secondaires tempérées, combinée à une importante disparition de forêts primaires et concentrée dans les pays tropicaux, avec de grandes disparités entre pays et selon les années. Lomborg minimise l'importance de cette dérive sur la base d'un hypothétique transfert des pressions vers les forêts secondaires, avec comme conséquence une relative protection des forêts primaires rémanentes.

Ce dernier argument se prolonge au travers de l'évolution souhaitable, selon Lomborg, du mode de gestion des forêts primaires dans les pays en voie de développement (PVD). Pour lui, la réappropriation par les PVD de leurs ressources en bois est un élément-clé de la croissance économique dans ces pays, qui conduirait naturellement à une diminution de la pression sur les forêts primaires par une utilisation plus raisonnée du combustible bois (valorisation plus efficiente, transfert vers d'autres types de carburant pour satisfaire les besoins en énergie) et la mise en place d'une agriculture plus performante (moins liée au défrichage). Cette évolution n'est pas utopique $^{9}$ mais néanmoins une fois de plus simplificatrice : Lomborg néglige par exemple la nécessité de réglementer les exportations de bois (tropicaux), à l'échelle de la planète, dans l'optique d'une gestion durable des ressources comme il donne à penser que tout ce qui est économiquement rationnel pourra effectivement être réalisé, sans tenir compte pour autant de la complexité des situations concrètes.

\section{Croissance et qualité de l'air}

L'un des plus grands succès de la diminution des impacts environnementaux concerne la réduction de

\footnotetext{
${ }^{9}$ Comme le souligne Lomborg lui-même, les modalités de traités engageant des PVD vis-à-vis de la protection de leurs forêts primaires sont à l'étude actuellement.
} 
certaines émissions acidifiantes comme le $\mathrm{SO}_{2}$ et les $\mathrm{NO}_{x}$ dans les pays développés. Celles-ci ont bénéficié des mesures d'économie d'énergie et de la centralisation de solutions technologiques économiquement abordables : modifications de combustibles (désulfurés) ou de procédés (brûleurs «low $\mathrm{NO}_{x} »$ ), pose de filtres sur de grandes installations émettrices, délocalisation d'industries lourdes et changement de sources d'énergie (gaz naturel et nucléaire ${ }^{10}$ ), pour certaines d'entres elles, motivées par des raisons stratégiques. En outre, la pollution par le $\mathrm{SO}_{2}$ est visible, respirable et entraîne des impacts immédiats sur la santé, ce qui a fait pression pour chercher des solutions depuis la révolution industrielle. On pouvait se contenter au début de construire des cheminées, mais les épisodes de smogs meurtriers (comme à Londres en 1952) ont finalement exigé des mesures plus radicales. Le problème de l'acidification n'est pas résolu pour autant, même dans les pays développés (en Europe, entre 7 et $36 \%$ des arbres sont endommagés suivant les pays), mais s'accroît en fait par le développement industriel rapide de certains pays (la Chine, par exemple).

Sur la base de l'évolution à Londres, et plus généralement en Europe et aux États-Unis, Lomborg présente les tendances de cette problématique comme reflétant un succès sans mélange. Pourtant au niveau mondial ce qui est bien son cadre de référence - les émissions ne cessent d'augmenter (Lefohn et al., 1999). Vu l'abondante littérature consultée, l'auteur ne pouvait ignorer cette croissance importante de la pollution dans certains pays en voie de développement.

À un niveau de calcul plus précis, penchons-nous encore sur la façon dont les données londoniennes ont été traitées par Lomborg, dont la conclusion est que «la pollution de l'air n'est pas un nouveau problème qui empire, mais un vieux problème qui ne fait que s'améliorer ». Il illustre son propos par un graphe (graphe 86), représentant une courbe en U inversé, de la qualité de l'air à Londres de 1585 à nos jours. D'après cette figure, la concentration de $\mathrm{SO}_{2}$ aurait crû jusqu'à près de $900 \mu \mathrm{g} / \mathrm{m}^{3}$ vers 1850, époque à partir de laquelle elle n'aurait cessé de baisser ${ }^{11}$. L'auteur cite notamment un scientifique renommé, Brimblecombe, mais nous apprend dans la note 1163 qu'il a dû «ajuster ces données par un facteur 4 pour les faire coïncider avec les données récentes observées ». Vérification faite, la valeur donnée par Brimblecombe (1977) vers 1850 n'atteint pas $200 \mu \mathrm{g} / \mathrm{m}^{3}$. Dès lors, c'est

\footnotetext{
${ }^{10}$ La réduction des émissions atmosphériques se réalise au prix d'autres pollutions comme les déchets radioactifs, quand l'électricité d'origine nucléaire remplace en partie celle issue de combustibles carbonés.

11 Notons que ces valeurs " moyennes annuelles " grossièrement estimées dépassent très largement les normes actuelles européennes, édictées pour des valeurs de pointe $\left(350 \mu \mathrm{g} / \mathrm{m}^{3}\right.$ comme valeur horaire ne peut pas être dépassé plus de 24 fois en une année).
}

à partir de cette extrapolation simplifiée que Lomborg arrive à la conclusion que nous sommes revenus à des niveaux de pollution du Moyen Âge.

La courbe en question fait apparaître le $X X^{\mathrm{e}}$ siècle comme une période de nette amélioration de la qualité de l'air, contredisant ainsi la litanie : le développement économique spectaculaire des années 1960 se serait donc fait au bénéfice de l'environnement! Mais cette courbe en U inversé, retrouvée par Lomborg pour Londres, a aussi une portée encore plus large, dans la mesure où, si elle avait été vérifiée, elle viendrait à l'appui d'une théorie centrale implicite dans son livre : la diminution programmée des impacts environnementaux grâce aux progrès technologiques, après une phase d'accroissement initial. Cette théorie (non citée ici par Lomborg, mais reprise à l'occasion par la Banque mondiale ou l'OCDE) est dénommée «courbes environnementales de Kusnetz » (CEK) (du nom de l'économiste qui l'a appliquée à la relation entre PIB et inégalités ${ }^{12}$ ). Dans certains domaines, au premier plan desquels les émissions acidifiantes, et pour beaucoup de villes occidentales, on peut en effet observer ce type de courbe. Mais cette bonne nouvelle technologique doit être mise en regard de plusieurs éléments majeurs. D'abord, certaines méta-études plus poussées montrent que la CEK ne se vérifierait pas, même pour le $\mathrm{SO}_{2}$, et se révèle extrêmement sensible au choix des exemples considérés (Harbaugh et al., 2002). Plus fondamentalement, elle ne s'observe pas pour tous les problèmes environnementaux, loin de là, comme on le voit par exemple avec les émissions de gaz à effet de serre ou la production de déchets qui continuent généralement à croître avec le PNB. Ensuite, même si elle était vérifiée, elle ne démontrerait pas pour autant qu'il s'agit de la trajectoire la plus efficiente du développement ${ }^{13}$ : la croissance initiale de l'économie engendre des dommages et il n'est pas prouvé que les laisser se produire est préférable à agir pour les éviter. Enfin, cette évolution n'est pas une conséquence spontanée du développement économique et technologique comme le soutient Lomborg : elle nécessite une action correctrice de la société, et en premier lieu des pouvoirs publics ${ }^{14}$.

L'optimisme que Lomborg veut nous faire partager est typique des économistes néoclassiques (Keyfitz, 1994), alors qu'il est maintenant démontré que cette

\footnotetext{
12 Simon Kuznets avait estimé en 1955 qu'après une première croissance des inégalités en fonction du PNB, celles-ci décroissent après être passées par un maximum. Cette affirmation n'est d'ailleurs pas généralisable comme on le voit par l'accroissement des inégalités de revenus dans les pays développés ces dernières décennies.

13 Au contraire, selon F. Granja e Barros et al. (2002).

${ }^{14} C^{\prime}$ est d'ailleurs la critique de M. Grubb dans Science (déjà cité), dans son article au titre évocateur : «Relying on manna from Heaven ? : les progrès enregistrés ne viennent pas du ciel mais sont stimulés par les actions des pouvoirs publics.
} 
théorie ne peut, sans corrections importantes à tout le moins, résoudre les problèmes environnementaux ${ }^{15}$. Mais ici, à l'appui de cette théorie, il faut citer la figure capitale de référence pour l'entreprise «sceptique » de Lomborg, celle de l'économiste Julian Simon. Une citation de cet auteur est placée en exergue du livre, et toute la thèse de Lomborg sur le progrès économique autocorrecteur de ses impacts environnementaux, sur les dépenses inutilement élevées de corrections publiques, se trouve déjà chez Simon et ses élèves.

\section{Quelques enseignements}

La ligne de plus forte pente, lorsque l'on analyse le travail de Lomborg, conduit à se positionner «pour» ou «contre »sa thèse. Tout le propos a été construit pour entraîner ce type de position. Pourtant nous voudrions, malgré les biais considérables de l'ouvrage, tenter de relever le défi qu'il lance en réfutant la solidité scientifique de l'alarmisme environnementaliste. Ce qui précède montre que la thèse optimiste de Simon-Lomborg prête le flanc à des critiques majeures. Hélas, la qualité des résultats factuels comporte également des erreurs, involontaires ou volontaires. Néanmoins, croire le brûlot lomborgien neutralisé pour se réfugier dans les « certitudes environnementalistes », dans leur version « litanie», ne nous paraît pas non plus très attractif. Nous évoquerons donc, pour terminer, plusieurs questions pertinentes qui nous paraissent avoir été révélées crûment par cet épisode.

La première concerne les conséquences qui découlent de l'évaluation de questions de développement durable en prenant le niveau mondial pour référence prépondérante. Dire par exemple que les prises de poissons surexploitent peu (Lomborg) ou beaucoup (la litanie) les stocks globaux, c'est débattre en référence à ce niveau mondial, qui n'est pas le seul pertinent. Ainsi, les pêcheurs mauritaniens ou sénégalais pour qui les prises se raréfient, seront peu dédommagés d'apprendre qu'elles augmentent à quelques milliers de kilomètres de là (nous avons vu une agrégation du même type déjà avec le cas des forêts). Certes, l'entreprise internationale de pêche modifiera l'origine de sa marchandise, et le consommateur qui achète des poissons surgelés n'y verra guère de différence. Mais au niveau local - et local peut signifier régional, national -, les problèmes peuvent être graves et passer inaperçus dans ces approches globales. Sans compter que ces "stocks" de poissons (notion issue de l'économie) nous disent peu de choses sur la qualité de la faune et du milieu. Une autre erreur d'un type un peu différent consiste à mesurer la biodiversité de préférence par le nombre d'espèces éteintes au niveau mondial. Tant

\footnotetext{
15 Voir par exemple les travaux de Passet, Daly, etc. via la Société internationale d'écologie économique (http://www.euroecolecon.org).
}

que le tigre, par exemple, n'a pas disparu partout, ce n'est pas trop grave?

Très souvent aussi, se surimposant à cette approche mondiale de l'environnement et des ressources, on trouve des surestimations contestables de l'apport des régulations économiques. Lomborg explique par exemple qu'il faut diminuer les subsides à l'agriculture dans les PVD et que des agricultures plus performantes (il cite les ÉtatsUnis) pourraient fournir des denrées avec une meilleure efficacité globale ${ }^{16}$.

Mais une question n'est pas abordée ici : d'où viendront les ressources de la masse d'agriculteurs disposant de moins d'un dollar par jour pour payer ces importations? Ce raisonnement prenant pour acquis le monde comme un système global néglige toutes les contraintes pour rendre disponible une ressource ailleurs qu'en son lieu de production : transport, système social, distribution, agriculture comme mode de vie, etc. ${ }^{17}$.

Ce que l'approche mondiale tend à masquer, ce sont la répartition des problèmes et la diversité des processus. Autant d'éléments qui contredisent les «grands » principes dont sont friandes les déclarations officielles : «il faut une approche mondiale pour protéger l'environnement », «les ressources mondiales diminuent », « les subsides nuisent à l'environnement », etc. Lomborg n'est certainement pas seul dans cette approche mondiale, aujourd'hui très répandue. Il n'est pas seul non plus dans son recours majeur à l'estimation économique des dommages environnementaux, dont l'utilisation fonde la hiérarchisation des décisions correctrices. De nombreux rapports d'instances internationales les utilisent fréquemment, mais en les combinant à d'autres arguments et d'autres contraintes, alors que The Skeptical Environmentalist fait un usage massif et univoque de cette approche qui n'est logique et cohérente qu'en apparence. Lomborg néglige ainsi les imprécisions considérables inhérentes aux méthodes d'estimations coûts-bénéfices (notamment en ne comparant pas différentes études, alors que les conventions de calcul mènent à des résultats qui peuvent diverger fortement). Il utilise des modes simples de calcul économique en dehors de leurs limites de pertinence, par exemple quand les facteurs ou les effets sont biologiques, irréversibles ou non substituables, ou quand ils ne peuvent pas faire l'objet de préférences des agents économiques ${ }^{18}$. Il tend à confondre la théorie économique et la réalité, comme lorsqu'il estime que les conflits pour l'eau sont improbables car trop coûteux par rapport à la valeur

\footnotetext{
16 Or, si le marché concurrentiel mène à de bons résultats agrégatifs, il n'en assure pas la redistribution (Sen, 1999).

17 Sans compter que la diminution de l'encadrement des agriculteurs du Sud est l'un des facteurs responsables des mauvaises pratiques, de la surexploitation des sols, de la déficience des connaissances techniques, du manque d'engrais (Reed, 1999).

18 Voir Faucheux et Noël, 1995.
} 
d'échange de cette ressource. Il prône des hiérarchies de choix basées sur des résultats statistiques fragiles. Ainsi, dans la section "Weighing Risks », une classification des risques est présentée (selon une étude de 1979, Tableau 8) qui conduit à revoir l'attribution des ressources en fonction du coût statistique de chacun d'eux en termes de vies humaines. Outre les différents problèmes déjà signalés (grandes imprécisions dans les évaluations, méconnaissance des processus à l'œuvre et de leur correction sur le terrain), il faut ajouter que le critère de coût (montré dans le Tableau 9 de son ouvrage) ne peut être le seul pour prendre ce type de décisions, en particulier en santé publique ${ }^{19}$. Dans le cas contraire, il faudrait cesser par exemple de soigner des personnes âgées ou très malades.

La vertu du livre de Lomborg, dont l'honnêteté sur plusieurs points a été contestée, pourrait être néanmoins de pousser à repenser les choix des approches et des cadrages qui peuvent être si déterminants. On a besoin de meilleures évaluations de l'environnement dans sa « complexité » (ce lieu commun ressassé), d'une communication avec les sphères de décision (ce que ce livre a si bien réussi), et enfin de s'interroger sur le réalisme des principes passe-partout.

En somme, ce livre nous appelle à mieux préciser les problèmes dont nous parlons et que nous voulons résoudre, au présent et aussi au futur. L'un de ses points les plus forts est qu'en effet il y a eu des progrès dans l'état de l'environnement, pour plusieurs problématiques, dans les pays riches en tout cas. On ne peut simplement les passer sous silence ${ }^{20}$. Sur le plan psychologique ou médiatique, The Skeptical Environmentalist nous amène à nous poser la question de savoir pourquoi l'écologie se marie souvent avec le catastrophisme, et comment elle pourrait devenir assez mature pour recevoir un traitement différencié dans la conscience du $X X^{e}$ siècle (question à laquelle, hélas, Lomborg ne répond pas, participant au contraire au même phénomène, en ayant juste inversé le miroir).

En outre, Lomborg fait sienne cette vision du développement durable où des problèmes écologiques sont

\footnotetext{
${ }^{19}$ La méthode «coûts-bénéfices » prônée par Lomborg implique de donner une valeur monétaire à la vie humaine. À la question éthique fondamentale : "comment peut-on donner une valeur monétaire à la vie humaine? ", les économistes répondent par des estimations éparses, contradictoires, et globalement proportionnelles au revenu monétaire, ce qui tend à donner plus d'importance à la vie d'un riche qu'à celle d'un pauvre. Ce qui est en contradiction avec les déclarations humanistes de Lomborg sur la nécessité d'aider les plus pauvres.

${ }^{20}$ Pas plus évidemment que l'on ne doit se laisser distraire par des questions résolues. Il est des problèmes environnementaux pour lesquels les solutions envisagées n'ont pas encore réussi à inverser les tendances, comme la perte de biodiversité, les émissions mondiales de $\mathrm{CO}_{2}$, la dissémination de substances nocives, l'érosion des sols, etc.
}

mis en balance avec des problèmes sociaux, qui, dans les pays pauvres, sont en effet d'une gravité extrême.

Légitime, cette approche doit cependant se garder d'adopter une série de biais déjà rencontrés. Ainsi, on n'a pas attendu The Skeptical Environmentalist pour savoir que «la plus grande priorité » qui devait être accordée, selon les vœux du Rapport Brundtland, aux «besoins essentiels » n'est toujours pas mise en œuvre.

Dans le « développement » actuel, cette plus grande priorité est en fait accordée à la demande solvable. Lomborg consent également parfois à sacrifier les plus faibles dans l'intérêt " général ", comme on l'a vu plus haut dans le cas de l'agriculture. Par contre, s'il est cohérent avec le principe de non-intervention de l'État dans l'économie, quand il préconise de supprimer les budgets environnementaux, il ne l'est plus quand il suggère de les transférer vers les pays en voie de développement. Ce n'est évidemment pas le transfert très hypothétique de budgets environnementaux vers ces pays qui va changer grand-chose aux problèmes des plus pauvres. Encore moins dans une vision où le marché se voit attribuer le rôle de régulateur prépondérant.

\section{Références}

Allègre, C., 2002. Écologie - L'état de la planète ou la conscience sans science, L'Express, 22 août.

Bongaarts, J., 2002. Population: ignoring its impact, Scientific American, 286, 6769.

Brimblecombe, P., 1977. London air pollution, 1500-1900, Atmospheric Environment, 11, 1157-1162.

Budiansky, S., 2002. Divisionary tactics in environmental debate, Nature, 415, 364.

Burgman, M., 2002. The Skeptical Environmentalist. Measuring the Real State of the World, Austral Ecology, 27, 238-239.

Chadwick, M.J., 2002. The Skeptical Environmentalist. Measuring the Real State of the World, Climate Policy, 2, 259-261.

Cole, M.A., 2003. Environmental optimists, environmental pessimists and the real state of the world: An article examining The skeptical environmentalist : Measuring the real state of the world by Bjorn Lomborg, The Economic Journal, 113 (June), 362-380.

Czech, B., 2000. Julian Simon Redux. Conservation Biology, 16, 2, April 2002, 570-571.

Dagg, J.L., 2003. The Skeptical Environmentalist and the Lomborg Affair, Environmental Science E Policy, 6, 4, sous presse.

Ege, C., Lind Christiansen, J., 2002. Sceptical Questions and Sustainable Answers, Copenhaguen, Danish Ecological Council.

Faucheux, S., Noël, J.F., 1995. Économie des ressources naturelles et de l'environnement, Paris, Armand Colin.

Godard, O., 2002. Bjørn Lomborg ou Tintin au pays de l'écologie? Une revue critique du livre de Bjørn Lomborg, The Skeptical Environmentalist, Politique étrangère, IFRI.

Granja e Barros, F., Mendoça, A.F., Nogueira, J.M., 2002. Poverty and Environmental Degradation : the Kuznets Environmental Curve for the Brazilian Case, Department of Economics Working paper, 267, University of Brasilia. 
Grubb, M., 2001. Relying on Manna from Heaven, Science, 294, 1285-1286.

Harbaugh, W.T., Levinson, A. and Wilson, D.M., 2002. Reexamining the Empirical Evidence for an Environmental Kuznets Curve, The Review of Economics and Statistics, July (http://harbaugh.uoregon.edu/index.htm\#Research).

Holdren, J., 2002. Energy : asking the wrong questions, Scientific American, 286, 6567.

Keyfitz, N., 1994. Croissance démographique : qui peut en évaluer les limites?, La Recherche, 264, 25.

Lefohn, A.S., Husar, J.D. and Husar, R.B., 1999. Estimating Historical Anthropogenic Global Sulfur Emission Patterns for the Period 1850-1990, Atmospheric Environment, 33, 3425-2444 (données disponibles sur http://www.uea.ac.uk/ e490/su/sulfur.htm).

Lovejoy, T., 2002. Biodiversity: dismissing scientific process, Scientific American, 286, 1, 6971.
Moomaw, W.R., 2002. Lomborg's The Skeptical Environmentalist : Refuting a Scientific Model without Science, Conservation Biology, 16, 4, 861-862.

Orr, D.W., 2002. The Labor of Sisyphus, Conservation Biology, 16, 4, 857-860.

Pauly, D., 2002. The Skeptical Environmentalist. Measuring the Real State of the World, Fisheries, 3, 360-365.

Pielke, Jr., R.A., 2002. Policy, politics and perspective, Nature, 416, 367-368.

Pimm, S. and Harvey, J., 2001. No need to worry about the Future, Nature, 414, 149-150.

Reed, D. (Ed.) 1999. Ajustement structurel, environnement et développement durable, Paris, L'Harmattan.

Ridley, M., 2002. The profits of doom, Spectator, 288, 1011.

Schneider, S., 2002. Global warming : neglecting the complexities, Scientific American, 286, 6265.

Sen, A., 1999. Un nouveau modèle économique. Développement, justice, liberté, Paris, Odile Jacob. 